\title{
LQR distributed cooperative control of a formation of low-speed experimental UAVs
}

\author{
Ivana Tomić \\ School of Mathematics, Computer \\ Science and Engineering, \\ City University, London, UK \\ Email: Ivana.Tomic.1@city.ac.uk
}

\author{
Efstathios Milonidis \\ School of Mathematics, Computer \\ Science and Engineering, \\ City University, London, UK \\ Email: E.Milonidis@city.ac.uk
}

\author{
George D. Halikias \\ School of Mathematics, Computer \\ Science and Engineering, \\ City University, London, UK \\ Email: G.Halikias@city.ac.uk
}

\begin{abstract}
The paper presents a cooperative scheme for controlling arbitrary formations of low speed experimental UAVs based on a distributed LQR design methodology. Each UAV acts as an independent agent in the formation and its dynamics are described by a 6-DOF (degrees of freedom) nonlinear model, linearized for control design purposes around an operating point corresponding to straight flight conditions. It is shown that the proposed controller stabilizes the overall formation and can control effectively the nonlinear multi-agent system. Formation control is extended to reference tracking by introducing integral action in the controller. It is shown via numerous simulations that the system is robust to environmental disturbances such as nonuniform wind gusts acting on a formation of four UAVs and to the loss of communication between two neighbouring UAVs.
\end{abstract}

\section{INTRODUCTION}

Cooperative control of multi-vehicle systems has been an active research area in recent years due to its broad spectrum of applications, such as surveillance and area exploration [1], load transportation [2], intelligent highways [3], formation control [4]-[6], etc. These systems are formed from a large number of dynamical subsystems, such as unmanned aerial vehicles (UAVs), unmanned ground vehicles (UGVs), unmanned underwater vehicles (UUVs), satellites or mobile robots.

UAVs are autonomous flying vehicles equipped with sensing devices that have many potential military and civil applications, but are also of great scientific significance in academic research. The problem of coordinating a predefined multivehicle formation while moving in space, known as formation control, is a topic of considerable interest to the control community. This is mainly due to its advantages over conventional systems, such as a reduction of system cost, an increase in the efficiency and robustness of the system, etc., see e.g. [7].

Linear Quadratic Regulator (LQR) theory has been widely used in multi-agent systems' control due to its guaranteed robustness properties, see e.g. [8]-[10]. Literature tends to favour distributed LQR-based control designs, as centralized solutions become infeasible as the number of subsystems and the distance between them increases [11].

The problem of controlling a formation of interacting and cooperating identical subsystems was first considered in [5]; in this work the communication topology of the network was modelled using graph theory. Further, the formation stability analysis for a given communication topology was proposed.
In [12] the framework was extended to the robust formation control method for an arbitrary communication topology and any number of subsystems, whereas previous design methods were adequate only for undirected communication networks. Also, the framework was used later in [13]-[15] to establish different distributed LQR-based control designs for controlling the formation.

In the present work we use the distributed LQR design strategy for uncoupled continuous-time multi-agent systems that has been introduced in [13] for controlling the multiagent formation. The authors proposed an approach which leads to an elegant and powerful result: the synthesis of stabilizing distributed control laws can be obtained by using a simple local LQR problem whose size depends on the maximum vertex degree of the graph. Compared to [13], where the model with double-integrator dynamics was analyzed, we consider a formation of $N$ identical low-speed experimental UAVs, known as X-RAE1, that can communicate with each other to achieve the common goal. Individual subsystems are described by 6-DOF (degrees of freedom) nonlinear model, which is linearized at certain operating points for the straight level flight dynamics. The graph-theory framework proposed in [5] is used to model the communication network of the subsystems. It is shown that the proposed controller stabilizes the system. Also, it can be used to control effectively the nonlinear multi-agent system for a standard set of initial conditions. Formation control is extended to reference tracking by introducing integral action in the controller. Further, it is shown that both multi-agent systems, linear and nonlinear, are robust to environmental disturbances such as nonuniform wind profiles for a formation of UAVs and to the loss of communication between any two agents.

The remainder of this paper is organized as follows. In Section II, notation and brief preliminaries on algebraic graph theory are presented. In Section III the nonlinear and linear models of a single X-RAE1 are briefly described. Section IV presents the procedure for modeling the multi-UAV system together with the distributed controller design procedure for this system. The proposed design is extended to accommodate integral action in the controller to achieve asymptotic tracking to step commands and asymptotic rejection of impulsive disturbances modeling wind gusts. Simulation results 
are presented and discussed in Section V. Finally, the paper's conclusions appear in Section VI.

\section{PRELIMINARIES}

\section{A. Notation and Definitions}

Let $I_{n}$ denote the identity matrix of dimension $n$, $I_{n} \in \mathbb{R}^{n \times n}$. Let $M^{T}$ and $\boldsymbol{a}^{T}$ denote, respectively, the transpose of matrix $M$ and the transpose of column vector $\boldsymbol{a}=\left[a_{1}, \ldots, a_{n}\right]^{T} . A \otimes B$ denotes the Kronecker product of $A$ and $B$. Let $A \in \mathbb{R}^{m \times n}$ and $B \in \mathbb{R}^{p \times q}$, then:

$$
A \otimes B=\left(\begin{array}{cccc}
a_{11} B & a_{12} B & \ldots & a_{1 n} B \\
\vdots & \vdots & \ddots & \vdots \\
a_{m 1} B & a_{m 2} B & \ldots & a_{m n} B
\end{array}\right) \in \mathbb{R}^{m p \times n q} .
$$

A matrix $M \in \mathbb{R}^{n \times n}$ is called stable or Hurwitz if all its eigenvalues have negative real part, i.e. $\mathcal{S}(M) \subseteq \mathbb{C}_{-}$.

\section{B. Graph Theory Preliminaries}

Let $\mathcal{G}=(\mathcal{V}, \mathcal{E})$ be an undirected graph with the set of nodes (or vertices), $\mathcal{V}=\{1,2, \ldots, N\}$, and the set of edges $\mathcal{E} \subseteq$ $\mathcal{V} \times \mathcal{V}, \mathcal{E} \subseteq\{(i, j): i, j \in \mathcal{V}, j \neq i\}$. If $i, j \in \mathcal{V}$ and $(i, j) \in \mathcal{E}$, then $i$ and $j$ are said to be adjacent (or neighbors) which is denoted as $i \sim j$. For an undirected graph the communication between two nodes (or agents) is bidirectional and we assume that there is no edge from a node to itself (i.e. no self loops). The number of neighboring nodes, $d_{i}$ for $i=1,2, \ldots, N$, is called the degree or valency of a node. Let $d_{\max }(\mathcal{G})$ denote the maximum node degree of the graph $\mathcal{G}$. Any undirected graph can be represented by its adjacency matrix, $\mathbf{A}(\mathcal{G})$. Let $\mathbf{A}_{i, j} \in \mathbb{R}$ be the $(i, j)$ element of $\mathbf{A}(\mathcal{G})$, then $\mathbf{A}_{i, i}=0, \forall i=$ $1,2, \ldots, N$, and

$$
\mathbf{A}_{i, j}=\left\{\begin{array}{l}
0 \text { if }(i, j) \notin \mathcal{E} \forall i, j=1,2, \ldots, N, \quad i \neq j, \\
1 \text { if }(i, j) \in \mathcal{E} \forall i, j=1,2, \ldots, N, \quad i \neq j .
\end{array}\right.
$$

\section{DYNAMICAL MODEL OF X-RAE1}

\section{A. Nonlinear Model}

The 6-DOF equations of motion of X-RAE1 with respect to the body-fixed axes are:

1) Translational equations of motion

$$
\begin{aligned}
\dot{U}= & R V-Q W-g \sin \Theta \\
& +\left[\bar{q} S\left(C_{L} \sin \alpha-C_{D} \cos \alpha\right)+T\right] / m \\
\dot{V}= & P W-R U+g \cos \Theta \sin \Phi)+\left(\bar{q} S C_{y}\right) / m \\
\dot{W}= & Q U-P V+g \cos \Theta \cos \Phi \\
& +\left[\bar{q} S\left(-C_{L} \cos \alpha-C_{D} \sin \alpha\right)\right] / m
\end{aligned}
$$

2) Rotational equations of motion

$$
\begin{aligned}
\dot{P} I_{x}-\dot{R} I_{x z}= & Q R\left(I_{y}-I_{z}\right)+P Q I_{x z}+\bar{q} S b C_{l} \\
\dot{Q} I_{y}= & P R\left(I_{z}-I_{x}\right)-\left(P^{2}-R^{2}\right) I_{x z}+\bar{q} S c C_{m}+ \\
& \bar{q} S\left(C_{L} \sin \alpha-C_{D} \cos \alpha\right) h_{0}+T e_{T} \\
\dot{R} I_{z}-\dot{P} I_{x z}= & P Q\left(I_{x}-I_{y}\right)+Q R I_{x z}+\bar{q} S c C_{n}
\end{aligned}
$$

where
- $U, V, W$ are forward, side and downward velocities along the $x, y$ and $z$ body axes, respectively;

- $P, Q, R$ are roll, pitch and yaw angular velocities around the $x, y$ and $z$ body axes, respectively;

- $\Phi, \Theta, \Psi$ are roll, pitch and yaw angles;

- $T$ is the thrust;

- $C_{L}, C_{D}, C_{y}$ are lift, drag and side force coefficients;

- $C_{l}, C_{m}, C_{n}$ are rolling, pitching and yawing moment coefficients;

- $I_{x}, I_{y}$ and $I_{z}$ are moments of inertia about the corresponding body axes;

- $I_{x z}$ is the product of inertia. As the aircraft has the $x z$ plane as a plane of symmetry $I_{x y}=I_{y z}=0$;

- $\alpha, \bar{q}$ are the angle of attack and the dynamic pressure;

- $m, g, S, e_{T}, h_{0}, b$ and $c$ are known parameters.

Additionally, we have three differential equations that are used to relate the body system rates $P, Q$ and $R$ to the three Euler rates $\dot{\Phi}, \dot{\Theta}$ and $\dot{\Psi}$. These are:

$$
\begin{aligned}
\dot{\Phi} & =P+Q \tan \Theta \sin \Phi+R \tan \Theta \cos \Phi \\
\dot{\Theta} & =Q \cos \Phi-R \sin \theta \\
\dot{\Psi} & =(R \cos \Phi+Q \sin \Phi) / \cos \Theta .
\end{aligned}
$$

For more details see [16].

\section{B. Linearized Model}

In order to design a linear controller, the nonlinear model is linearized and decomposed into two motions, longitudinal and lateral, by assuming small perturbations around the operating point. For a straight, steady, symmetric and horizontal flight at a constant velocity $V_{T_{0}}=30 \mathrm{~m} / \mathrm{s}$, the following trimmed values are considered:

$$
\begin{aligned}
U_{0} & =V_{T_{0}} \cos \alpha_{0}, W_{0}=V_{T_{0}} \sin \alpha_{0}, \\
V_{0} & =P_{0}=Q_{0}=R_{0}=0, \\
\Theta_{0} & =\alpha_{0} \text { and } \Phi_{0}=\Psi_{0}=0 .
\end{aligned}
$$

Then, the state space longitudinal model

$$
\dot{\boldsymbol{x}}_{\boldsymbol{i}}=A \boldsymbol{x}_{\boldsymbol{i}}+B \boldsymbol{u}_{\boldsymbol{i}}, \quad \boldsymbol{x}_{\boldsymbol{i}}(0)=\boldsymbol{x}_{\boldsymbol{i 0}}
$$

where $\boldsymbol{x}_{\boldsymbol{i}}=\left[\begin{array}{llll}u & w & q & \theta\end{array}\right]^{T}, \boldsymbol{u}_{\boldsymbol{i}}=\left[\begin{array}{ll}\eta & \delta_{T}\end{array}\right]^{T}$ are the state and input vectors of the $i$ th system at time $t$, respectively, can be expressed as

$$
\begin{aligned}
{\left[\begin{array}{c}
\dot{u} \\
\dot{w} \\
\dot{q} \\
\dot{\theta}
\end{array}\right]=} & {\left[\begin{array}{cccc}
-0.142 & -0.227 & 2.493 & -9.771 \\
-1.033 & -4.476 & 28.639 & 0.837 \\
-0.042 & -2.744 & -15.351 & -0.134 \\
0 & 0 & 1 & 0
\end{array}\right]\left[\begin{array}{c}
u \\
w \\
q \\
\theta
\end{array}\right] } \\
& +\left[\begin{array}{cc}
-1.136 & 1.444 \\
-13.060 & 0 \\
-137.157 & -2.036 \\
0 & 0
\end{array}\right]\left[\begin{array}{c}
\eta \\
\delta_{T}
\end{array}\right]
\end{aligned}
$$

where $u, w, q$ and $\theta$ denote forward velocity, downward velocity, pitch angular velocity and pitch angle, respectively, while $\eta$ and $\delta_{T}$ are elevator deflection and throttle setting, respectively; the lower case notation denotes the deviation of each motion quantity from the trim value, i.e. $\mathrm{d} U=u$. For more details see [16]. 
For simulation purposes the disturbance in system (3) is introduced as an arbitrary impulse to the downward velocity variable $w$, which is equivalent to the presence of environmental disturbances such as nonuniform wind for a collection of agents.

\section{FORMATION MODELING AND CONTROLLER DESIGN}

\section{A. Modeling Multi-UAV System with LQR-based Control}

The collective dynamics of $N$ identical and decoupled dynamical agents can be described as:

$$
\dot{\boldsymbol{x}}(t)=A_{a} \boldsymbol{x}+B_{a} \boldsymbol{u}, \boldsymbol{x}(0)=\boldsymbol{x}_{0}
$$

where $\boldsymbol{x}(t)=\left[\boldsymbol{x}_{1}^{T}(t), \ldots, \boldsymbol{x}_{\boldsymbol{N}}^{T}(t)\right]^{T}$ and $\boldsymbol{u}(t)=$ $\left[\boldsymbol{u}_{\mathbf{1}}^{T}(t), \ldots, \boldsymbol{u}_{\boldsymbol{N}}^{T}(t)\right]^{T}$ are the vectors which collect the states and inputs of the $N$ systems, while $A_{a}=I_{N} \otimes A$ and $B_{a}=I_{N} \otimes B$, where $A$ and $B$ are defined as in (3).

The LQR problem for the system (4) is described through the cost function which contains terms for weighting the difference between $i$ th and $j$ th system states, as well as the $i$ th system state and input:

$$
\begin{array}{r}
J\left(\boldsymbol{u}(t), \boldsymbol{x}_{\mathbf{0}}\right)=\int_{0}^{\infty}\left(\sum_{i=1}^{N}\left(\boldsymbol{x}_{\boldsymbol{i}}(t)^{T} Q_{i i} \boldsymbol{x}_{\boldsymbol{i}}(t)+\boldsymbol{u}_{\boldsymbol{i}}(t)^{T} R_{i i} \boldsymbol{u}_{\boldsymbol{i}}(t)\right)+\right. \\
\left.\sum_{i=1}^{N} \sum_{\substack{j=1 \\
j>i}}^{N}\left(\left(\boldsymbol{x}_{\boldsymbol{i}}(t)-\boldsymbol{x}_{j}(t)\right)^{T} Q_{i j}\left(\boldsymbol{x}_{\boldsymbol{i}}(t)-\boldsymbol{x}_{j}(t)\right)\right)\right) \mathrm{d} t .
\end{array}
$$

which can be rewritten using the more compact notation:

$$
J\left(\boldsymbol{u}(t), \boldsymbol{x}_{\mathbf{0}}\right)=\int_{0}^{\infty}\left(\boldsymbol{x}(t)^{T} Q_{a} \boldsymbol{x}(t)+\boldsymbol{u}(t)^{T} R_{a} \boldsymbol{u}(t)\right) \mathrm{d} t
$$

where the matrices $Q_{a}$ and $R_{a}$ have the following structure:

$$
Q_{a}=\left(\begin{array}{cccc}
Q_{a_{11}} & Q_{a_{12}} & \ldots & Q_{a_{1 N}} \\
\vdots & \vdots & \ddots & \vdots \\
Q_{a_{N 1}} & Q_{a_{N 2}} & \ldots & Q_{a_{N N}}
\end{array}\right), R_{a}=I_{N} \otimes R
$$

with $Q_{a_{i i}}=\sum_{k=1}^{N} Q_{i k}$ for $i=1, \ldots, N, Q_{a_{i j}}=-Q_{i j}$ for $i, j=1, \ldots, N, i \neq j$, and $R_{i i}=R_{i i}^{T}>0, \forall i$. Further, $Q_{i i}=Q_{i i}^{T} \geq 0, \forall i$ and $Q_{i j}=Q_{i j}^{T}=Q_{j i} \geq 0 \forall i \neq j$.

We are assuming that the pairs $(A, B),\left(A_{a}, B_{a}\right)$ are stabilizable and the pairs $(A, C),\left(A_{a}, C_{a}\right)$ are observable for any $Q=Q^{T} \geq 0$ and $Q_{a}$ as in (6) (where $C^{T} C=Q$, $C_{a}^{T} C_{a}=Q_{a}$ ).

Then, for the given initial conditions, $\boldsymbol{x}_{\mathbf{0}}$, the control input $\boldsymbol{u}=-R_{a}^{-1} B_{a}^{T} P_{a} \boldsymbol{x}$ minimizes the cost function in (5) subject to $\dot{\boldsymbol{x}}(t)=A_{a} \boldsymbol{x}+B_{a} \boldsymbol{u}, \boldsymbol{x}(0)=\boldsymbol{x}_{\mathbf{0}}$. Also, $P_{a}$ is the symmetric positive definite stabilizing solution of the following (largescale) Algebraic Riccati Equation (ARE):

$$
A_{a}^{T} P_{a}+P_{a} A_{a}-P_{a} B_{a} R_{a}^{-1} B_{a}^{T} P_{a}+Q_{a}=0 .
$$

For $Q_{a_{i i}}=Q_{1} \forall i=1, \ldots, N$, and $Q_{a_{i j}}=Q_{2} \forall i=$ $1, \ldots, N, i \neq j P_{a}$ has the structure:

$$
P_{a}=\left(\begin{array}{cccc}
P_{a_{11}} & P_{a_{12}} & \ldots & P_{a_{12}} \\
P_{a_{12}} & P_{a_{11}} & \ldots & P_{a_{12}} \\
\vdots & \vdots & \ddots & \vdots \\
P_{a_{12}} & \ldots & \ldots & P_{a_{11}}
\end{array}\right)
$$

with $P_{a_{11}}=P-(N-1) P_{a_{12}}$ where $P \in \mathbb{R}^{n \times n}$ is the symmetric positive definite solution of the ARE:

$$
A^{T} P+P A-P B R^{-1} B^{T} P+Q_{1}=0 .
$$

Further, the same structure of diagonal and off-diagonal blocks will be preserved in the gain matrix $K_{a}$, as $K_{a}=R_{a}^{-1} B^{T} P_{a}$. For more details and proofs see [13].

\section{B. Distributed Controller Design Approach}

Consider the multi-agent system composed of $N_{d}$ identical and decoupled agents described as:

$$
\dot{\tilde{\boldsymbol{x}}}(t)=\tilde{A} \tilde{\boldsymbol{x}}+\tilde{B} \tilde{\boldsymbol{u}}, \quad \tilde{\boldsymbol{x}}(0)=\tilde{\boldsymbol{x}}_{0}
$$

where $\tilde{\boldsymbol{x}}(t)$ and $\tilde{\boldsymbol{u}}(t)$ are the states and inputs of the $N_{d}$ systems, while $\tilde{A}=I_{N_{d}} \otimes A$ and $\tilde{B}=I_{N_{d}} \otimes B$, where $A$ and $B$ are defined as in (3). Systems (4) and (10) differ only in the number of subsystems. The distributed optimal control problem is given in [13], but instead of solving NPhard problem, the suboptimal distributed design procedure is given next.

Theorem 4.1: [13] For the system in (10), with cost function:

$$
J\left(\tilde{\boldsymbol{u}}(t), \tilde{\boldsymbol{x}}_{0}\right)=\int_{0}^{\infty}\left(\tilde{\boldsymbol{x}}(t)^{T} \tilde{Q} \tilde{\boldsymbol{x}}(t)+\tilde{\boldsymbol{u}}(t)^{T} \tilde{R} \tilde{\boldsymbol{u}}(t)\right) \mathrm{d} t
$$

where $\tilde{Q}=\tilde{Q}^{T} \geq 0, \tilde{R}=\tilde{R}^{T}>0$ and $\tilde{Q}$ is structured as: $\tilde{Q}_{i i}=\tilde{Q}_{1}$ for all $i=1, \ldots, N$ and $\tilde{Q}_{i j}=\tilde{Q}_{2}$ for all $j=1, \ldots, N, i \neq j$, the gain matrix can be constructed as

$$
\tilde{K}=I_{N_{d}} \otimes R^{-1} B^{T} P-M \otimes R^{-1} B^{T} P_{a_{12}}
$$

where $P$ is the symmetric positive definite solution to the single agent LQR problem in (9) and $P_{a_{12}}$ represents the off-diagonal blocks of the solution $P_{a}$ in (7) for $N_{\min }=$ $d_{\max }(\mathcal{G})+1$ agents.

Matrix $M$ reflects the structure of the graph $\mathcal{G}$ and is given by $M=a I_{N_{d}}-b \mathbf{A}(\mathcal{G}), b \geq 0$ where $\mathbf{A}(\mathcal{G})$ is the adjacency matrix. Also, $a$ and $b$ have to satisfy $a-b d_{\max } \geq 0$ which follows from the gain margin properties of the proposed design. Then, the closed loop system:

$$
\tilde{A_{c l}}=\tilde{A}-\tilde{B} \tilde{K}=I_{N_{d}} \otimes A+\left(I_{N_{d}} \otimes B\right) \tilde{K}
$$

will be asymptotically stable and $\tilde{P}$ is the unique solution of the following Lyapunov equation:

$$
\tilde{A}_{c l}^{T} \tilde{P}+\tilde{P} \tilde{A}_{c l}+\tilde{Q}+\tilde{K}^{T} \tilde{R} \tilde{K}=0 .
$$

\section{Proof 4.1.1: See [13]}

\section{Integral Control}

The distributed LQR controller proposed in Section IV-B provides good performance for regulation tasks (as shown in Section V), but is not able to ensure the asymptotic tracking of step references due to the absence of integral action in the controller. To this end, the state-space system in (3) is 
augmented by an error vector $\boldsymbol{z} \in \mathbb{R}^{p}$, such that $\dot{\boldsymbol{z}}=C \boldsymbol{x}$. Then, distributed system with integral action is given by

$$
\begin{aligned}
\dot{\tilde{\boldsymbol{x}}}_{I} & =\tilde{A}_{I} \tilde{\boldsymbol{x}}_{I}+\tilde{B}_{I} \tilde{\boldsymbol{u}}_{I}, \quad \tilde{\boldsymbol{x}}_{I}(0)=\tilde{\boldsymbol{x}}_{I_{0}} \\
\tilde{\boldsymbol{y}}_{I} & =\tilde{C}_{I} \tilde{\boldsymbol{x}}_{I}
\end{aligned}
$$

where $\tilde{\boldsymbol{x}}_{I}=\left[\begin{array}{cc}\boldsymbol{x} & \boldsymbol{z}\end{array}\right]^{T}, \tilde{A}_{I}=\left[\begin{array}{cc}A & 0 \\ C & 0\end{array}\right], \tilde{B}_{I}=\left[\begin{array}{c}B \\ 0\end{array}\right]$ and $\tilde{C}_{I}=\left[\begin{array}{ll}C & 0\end{array}\right]$ in which $C$ is the output matrix. By solving the (large-scale) ARE:

$$
\tilde{A}_{I}^{T} \tilde{P}_{I}+\tilde{P}_{I} \tilde{A}_{I}-\tilde{P}_{I} \tilde{B}_{I} \tilde{R}_{I}^{-1} \tilde{B}_{I}^{T} \tilde{P}_{I}+\tilde{Q}_{I}=0
$$

for $\tilde{Q}_{I}=\left[\begin{array}{cc}\tilde{Q} & 0 \\ 0 & \gamma I\end{array}\right]$ and $\tilde{R}_{I}=\rho I$, we get the stabilizing solution $\tilde{P}_{I}$ ( $\gamma$ and $\rho$ are given positive constants). Furthermore, by using the gain matrix $\tilde{K}_{I C}=\tilde{R}_{I}^{-1} \tilde{B}_{I}^{T} \tilde{P}_{I}$ we define the control input as

$$
\tilde{\boldsymbol{u}}_{I}=-\tilde{K}_{I C} \tilde{\boldsymbol{x}}_{I}=\left[\begin{array}{ll}
K_{P} & K_{I}
\end{array}\right]\left[\begin{array}{l}
\boldsymbol{x} \\
\boldsymbol{z}
\end{array}\right]
$$

where the matrices $K_{P}$ and $K_{I}$ are the proportional state feedback gain and the integral output gain, respectively. The block diagram of the augmented closed-loop system with the integral control is given in Fig. 1.

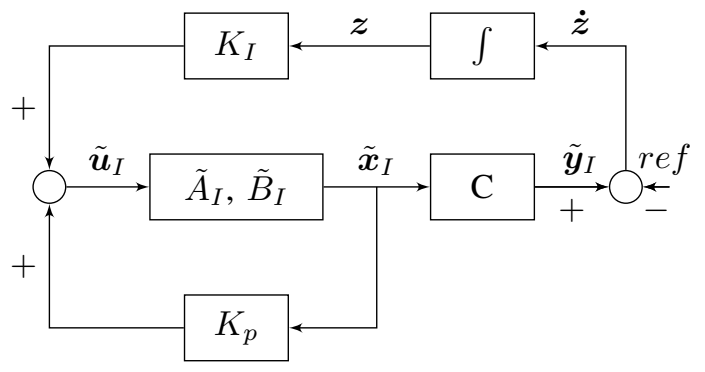

Fig. 1. Closed-loop distributed system with the integral control

\section{Simulation Results}

Consider a network of four dynamically decoupled XRAE1s moving in a plane, whose individual dynamics is linear and described in (3). The interconnection structure is depicted in Figure 2.

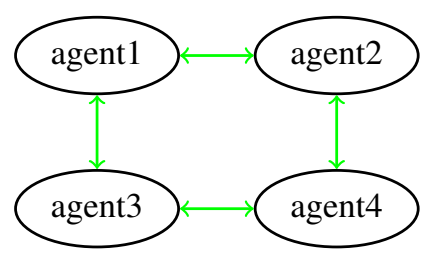

Fig. 2. The interconnection structure

The maximum vertex degree of the interconnection graph is 2, thus the size of LQR problem in (7) that has to be solved to design a stabilizing distributed controller is $N_{\min }=3$.
Then, we define the distributed LQR problem for a formation in Figure 2 as

$$
\min _{\tilde{K}} \tilde{J}\left(\tilde{\boldsymbol{u}}(t), \tilde{\boldsymbol{x}}_{0}\right) \quad \text { subj. to } \dot{\tilde{\boldsymbol{x}}}=\tilde{A} \tilde{\boldsymbol{x}}+\tilde{B} \tilde{\boldsymbol{u}}, \tilde{\boldsymbol{x}}(0)=\tilde{\boldsymbol{x}}_{0}
$$

where $\tilde{A}=I_{3} \otimes A$ and $\tilde{B}=I_{3} \otimes B$, and $A$ and $B$ are as in (3). The cost function $\tilde{J}\left(\tilde{\boldsymbol{u}}(t), \tilde{\boldsymbol{x}}_{0}\right)$ is as in (11) with the weighting matrices $\tilde{Q}$ and $\tilde{R}$, which are structured as: $\tilde{Q}_{i i}=\operatorname{diag}(0.3,3,0.15,3)$ for $i=1, \ldots, N_{m i n}$, $\tilde{Q}_{i j}=\operatorname{diag}(-0.1,-1,-0.05,-1)$ for $i, j=1, \ldots, N_{\text {min }}$ and $i \neq j$, and $\tilde{R}=I_{3} \otimes R$ with $R=10 I_{2}$.

The solution of the above minimum size LQR problem is of the following structure:

$$
P_{a}=\left[\begin{array}{lll}
P_{a_{11}} & P_{a_{12}} & P_{a_{12}} \\
P_{a_{12}} & P_{a_{11}} & P_{a_{12}} \\
P_{a_{12}} & P_{a_{12}} & P_{a_{11}}
\end{array}\right]
$$

Our control objective is to stabilize each individual agent moving on a plane by using the distributed gain matrix

$$
\tilde{K}=I_{4} \otimes R^{-1} B^{T} P-M \otimes R^{-1} B^{T} P_{a_{12}}
$$

where $P=P_{a_{11}}+2 P_{a_{12}}$ and $M=2 I_{4}-\boldsymbol{A}(\mathcal{G})$. Further, the adjacency matrix representing the graph is given by

$$
\boldsymbol{A}(\mathcal{G})=\left[\begin{array}{llll}
0 & 1 & 1 & 0 \\
1 & 0 & 0 & 1 \\
1 & 0 & 0 & 1 \\
0 & 1 & 1 & 0
\end{array}\right]
$$

Therefore, the first set of results is produced for a formation control by using the distributed controller in (19). Then, the model is extended to accommodate the reference tracking by using the distributed controller with integral control as described in Section IV-C with $\tilde{Q}_{I}=\left[\begin{array}{cc}\tilde{Q} & 0 \\ 0 & I\end{array}\right]$ and $\tilde{R}_{I}=10 I$.

In order to validate these two different control approaches a simulation environment is created for both models, linear and nonlinear, by using Matlab ${ }^{\circledR}$ and Simulink ${ }^{\circledR}$ [17]. Agents' movement is illustrated by examining the deviation from nominal velocity $V_{T_{0}}=30 \mathrm{~m} / \mathrm{s}$, which is the horizontal speed at which the model has been linearized. Further, the individual agents' vertical positions (i.e. heights) are depicted for both controllers.

The first simulation illustrates the height responses (i.e. deviation from the nominal height) of the each agent in Figure 2 in the presence of environmental disturbances for the case of linear dynamics. The disturbances are introduced as arbitrary impulses to the downward velocities of the four agents. Results are reproduced for two different controllers used to control the linear system, distributed LQR without integral action and distributed LQR with integral action and depicted in Figure 3. Similarly, Figure 4 depicts the deviation of each agent's velocity from the nominal velocity of $30 \mathrm{~m} / \mathrm{s}$ in the presence of environmental disturbances for the case of linear dynamics.

Figure 3 and Figure 4 demonstrate that both distributed controllers stabilize the formation. However, distributed controller without integral action was unable to ensure trajectory 

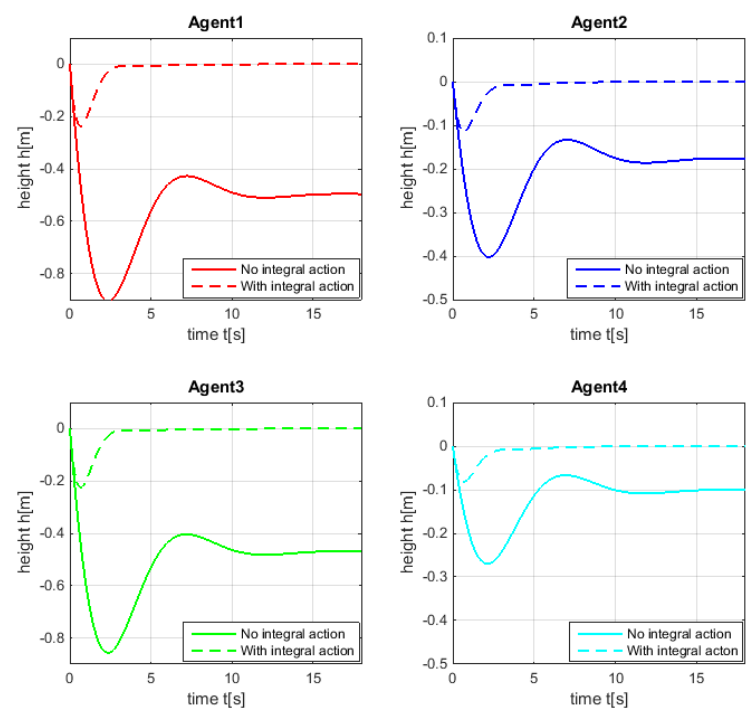

Fig. 3. Height responses of the linear LQR system controlled by the distributed controller with and without integral action in the presence of the impulse disturbance
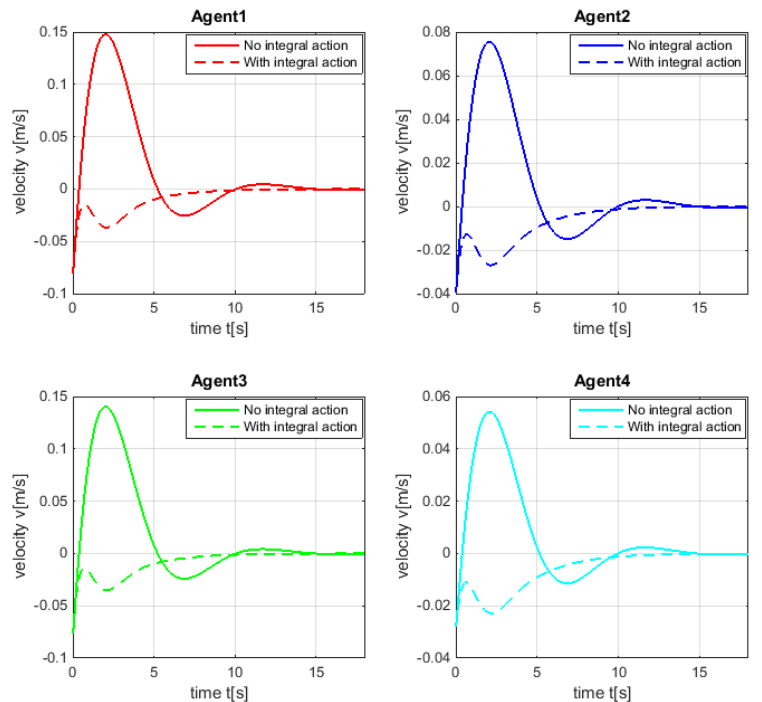

Fig. 4. Velocity responses of the linear LQR system controlled by the distributed controller with and without integral action in the presence of the impulse disturbance

tracking in the presence of external disturbances. The formation structure is lost as agents stabilize on different vertical positions. By incorporating integral action in the controller asymptotic tracking of step hight demands is achieved despite the presence of the impulsive disturbances on the downward velocity variables. Therefore, in the case of linear model agents were able to stabilize at requested locations with respect to their vertical positions. Please note that the formation structure is lost with respect to the horizontal agents' positions. This can be prevented, if required, by introducing integral action for horizontal regulation via the introduction of an additional state variable. Results are omitted due to space restrictions.

Next, the same set of results are reproduced for the nonlinear system using identical simulation parameters. These are given in Figure 5 and Figure 6. Despite strong nonlinearity in the
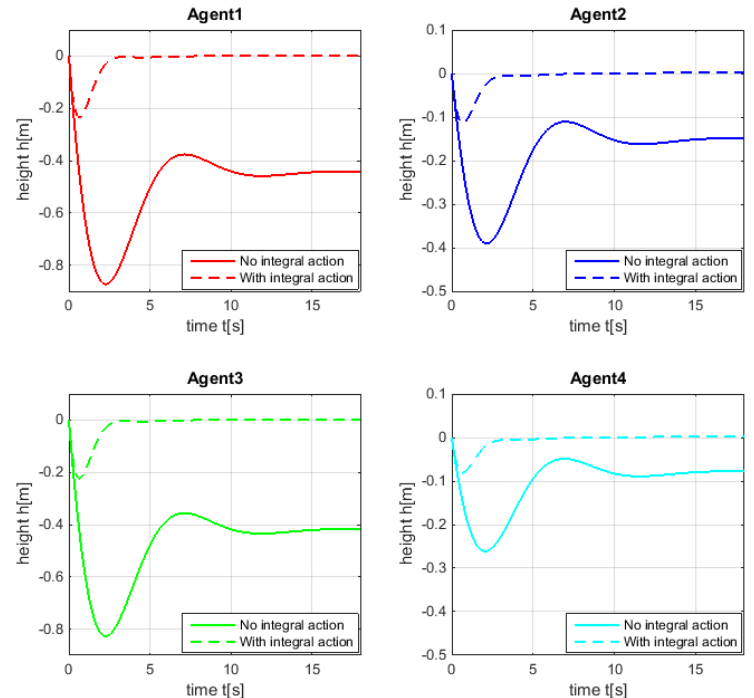

Fig. 5. Height responses of the nonlinear LQR system controlled by the distributed controller with and without integral action in the presence of the impulse disturbance
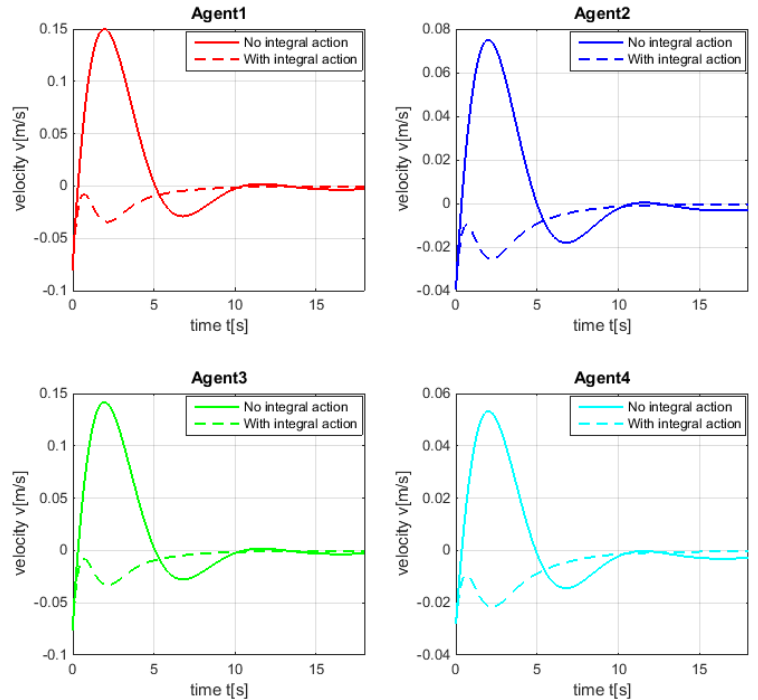

Fig. 6. Velocity responses of the nonlinear LQR system controlled by the distributed controller with and without integral action in the presence of the impulse disturbance

model, the controller was able to reproduce results that are closely related to those obtained in the linear case. However, the small deviation from the nominal velocity can be observed 
in Figure 6 for agent 2 and agent 4. The tracking of the reference signal is achieved only when distributed controller with integral action is used.

Finally, we considered the case when the communication between two agents is lost shortly after a disturbance impulse was applied. We assume the impulse disturbance to agent 1 at $t=9 \mathrm{~s}$ which is followed by the failure of link communication between agent 1 and agent 2 (in both directions) at $t=9.2 \mathrm{~s}$. Results are produced only for agent 1 in the case of nonlinear dynamics with integral action as this can be considered as the most critical case for the stability of the formation. Thus, the height and velocity responses of agent 1 are depicted in Figure 7.
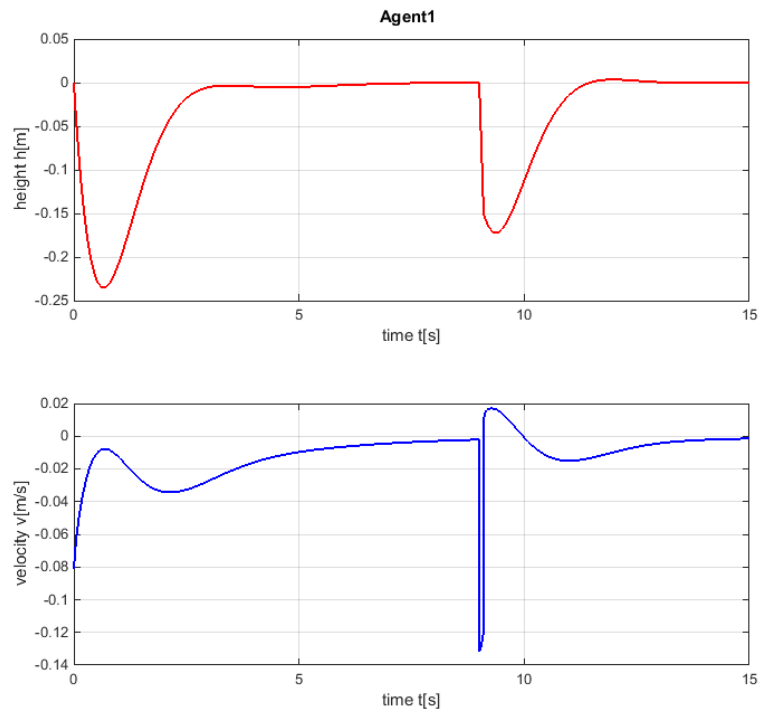

Fig. 7. Height and velocity responses of the nonlinear LQR system controlled by the distributed controller with integral action in the presence of an impulsive disturbance to agent 1 followed by the communication failure between agent 1 and agent 2

It can be seen that system stabilizes even in the case of link failure in the presence of disturbance as long as connectivity of network is preserved. These can be considered as the preliminary results that can be extended along various directions. Future work will investigate the size of disturbances that can be rejected, as well as the minimum time that system needs to recover from a communications failure before the next disturbance happens.

\section{CONCLUSIONS}

The paper has described a cooperative scheme for controlling a formation of low speed experimental UAVs based on distributed LQR control. The simulation results presented demonstrate the effectiveness of the method in dealing with nonlinear model dynamics, partial loss of communication between agents, rejection of external disturbances and asymptotic tracking requirements to step hight demands.

\section{REFERENCES}

[1] S. Waharte, N. Trigoni, N and S. J. Julier, Coordinated search with a swarm of UAVs, in 6th Annual IEEE Com. Society Conf. on Sensor, Mesh and Ad Hoc Com. and Networks Workshops, 2009. SECON Workshops '09., pp. 1-3, 2009.

[2] N. Michael, J. Fink, and V. Kumar, Cooperative manipulation and transportation with aerial robots, Autonomous Robots, vol. 30, pp. 73-86, 2011.

[3] J. G. Bender, An overview of systems studies of automated highway systems, IEEE Trans. Veh. Technol., vol. 40, pp. 82-99, 1991.

[4] A. Jadbabaie, J. Lin, and A. S. Morse, Coordination of groups of mobile autonomous agents using nearest neighbor rules, IEEE Trans. Automatic Control, vol. 48, no. 6, pp. 988-1001, 2003.

[5] J. A. Fax and R. M. Murray, Information flow and cooperative control of vehicle formations, IEEE Trans. Automatic Control, vol. 49, no. 9, pp. 1465-1476, 2004

[6] Y. Chen and Z. Wang, Formation control: a review and a new consideration, in Proc. the 2005 IEEE/RSJ International Conference on Intelligent Robots and Systems, pp. 3181-3186, 2005.

[7] D. J. Stilwell and B. E. Bishop, Platoons of underwater vehicles, IEEE Control Systems Magazine, vol. 20, no. 6, pp. 45-52, 2000.

[8] Y. C. Cao and W. Ren, Optimal linear-consensus algorithms: An LQR perspective. IEEE Trans. on Systems, Man, and Cybernetics-Part B: Cybernetics, vol. 40, no. 3, pp. 819-830, 2010.

[9] C. Langbort and V. Gupta, Minimal interconnection topology in distributed control design, SIAM J. Control Optim., vol. 48, no. 1, pp. $397-$ 413, 2009.

[10] P. Deshpande, P. Menon, C. Edwards, and I. Postlethwaite, A distributed control law with guaranteed LQR cost for identical dynamically coupled linear systems, in Proc. American Control Conference, pp. 5342-5347, 2011.

[11] P. Massioni and M. Verhagen, Distributed control for identical dynamically coupled systems: A decomposition approach, IEEE Trans. Automat. Contr., vol. 54, no. 1, pp. 124-135, 2009.

[12] A. P. Popov and H. Werner, A robust control approach to formation control, in Proc. 10th European Control Conf., Budapest, Hungary, pp. 4428-4433, 2009.

[13] F. Borrelli and T. Keviczky, Distributed LQR design for identical dynamically decoupled systems, IEEE Trans. Automat. Contr., vol. 53, no. 8, pp. 1901-1912, 2008.

[14] P. Massioni, T. Keviczky, and M. Verhaegen, New approaches to distributed control of satellite formation flying, in Proceedings of the 3rd Int. Symposium on Formation Flying, Missions and Technologies, Noordwijk, The Nederlands: ESA/ESTEC, pp. 22-27, 2008.

[15] D. Gu, A differential game approach to formation control, IEEE Tran. on Control Systems Technology, vol. 16, no. 1, pp. 85-93, 2008.

[16] I. Elgayar, Mathematical modelling, flight control system design and air flow control investigation for low speed UAVs, Ph.D. dissertation, City University London, London, 2013.

[17] Matlab and Simulink Release 2015a, The MathWorks, Inc., Natick, Massachusetts, United States. 